\title{
Hamiltonian Flow in Coulomb Gauge Yang-Mills Theory
}

\author{
Hugo Reinhardt* ${ }^{* \dagger}$ \\ Universität Tübingen \\ Institut für Theoretische Physik \\ Auf der Morgenstelle 14 \\ D-72076 Tübingen \\ Germany \\ E-mail: hugo.reinhardteuni-tuebingen.de
}

\section{Markus Leder ${ }^{a}$, Jan M. Pawlowski ${ }^{b}$ and Axel Weber ${ }^{c}$}

${ }^{a}$ Universität Tübingen, Institut für Theoretische Physik, Auf der Morgenstelle 14, D-72076

Tübingen, Germany

${ }^{b}$ Universität Heidelberg, Institut für Theoretische Physik, Philosophenweg 16, D-62910

Heidelberg, Germany

${ }^{c}$ Instituto de Física y Matemáticas, Universidad Michoacana de San Nicolás de Hidalgo, Edificio

C-3, Ciudad Universitaria, 58040 Morelia, Michoacán, Mexico

A new functional renormalization group equation for Hamiltonian Yang-Mills theory in Coulomb gauge is presented and solved for the static gluon and ghost propagators under the assumption of ghost dominance. The results are compared to those obtained in the variational approach.

The XXVIII International Symposium on Lattice Field Theory, Lattice2010

June 14-19, 2010

Villasimius, Italy

\footnotetext{
${ }^{*}$ Speaker.

† supported by DFG-Re856/6-3
} 


\section{Introduction}

Although lattice calculations have given us much insight into the non-perturbative regime of QCD, a thorough understanding of infrared phenomena like confinement and chiral symmetry breaking has not been achieved yet by lattice calculations as even the largest lattice calculations presently available do not yet probe sufficiently deep into the infrared. Hence, in recent years there have been many activities devoted to the non-perturbative studies of the infrared sector of continuum QCD. Among these are functional renormalization group (FRG) equations $[1,2]$ and the variational approach to the Hamiltonian formulation of QCD [3, 4]. Each approach has its own advantages and drawbacks and by combining these approaches one can expect to gain new insights into the theory, in particular into the non-perturbative regime.

My talk is devoted to the application of FRG flows to the Hamiltonian formulation of Yang-Mills theory as put forward in [1]. First, to motivate the FRG studies, I will briefly summarize some essential results of the variational Hamiltonian approach. Then I will give a short general introduction to FRG equations and present the new flow equations for Hamiltonian Yang-Mills theory in Coulomb gauge together with their solutions for the gluon and ghost propagators [1].

\section{Summary of the variational approach to Hamiltonian Yang-Mills theory}

In the variational approach to Yang-Mills theory in Coulomb gauge developed in our group one makes the following ansatz for the vacuum wave functional [4]:

$$
\psi=\frac{1}{\sqrt{\operatorname{Det}(-D \partial)}} \exp \left(-\frac{1}{2} \int A \omega A\right)
$$

where $\operatorname{Det}(-D \partial)$ is the Faddeev-Popov determinant and $\omega$ is a variational kernel, which is related to the static gluon propagator by

$$
\langle A A\rangle=(2 \omega)^{-1}
$$

and therefore has the meaning of the gluon energy. Minimizing the vacuum energy density with respect to $\omega(p)$ [4] one finds the gluon propagator [5], shown in Fig. 1 together with lattice data [6]. Both, the lattice data and the continuum solution can be fitted with Gribov's formula

$$
\omega(p)=\sqrt{\frac{M^{4}}{p^{2}}+p^{2}}
$$

with $M \simeq 860 \mathrm{MeV}$. At large momenta $\omega(p) \sim p$, in accordance with asymptotic freedom, whereas at small momenta $\omega(p) \sim 1 / p$, and this divergence shows the absence of gluons from the physical spectrum, also called gluon confinement. Fig. 2 shows the ghost form factor $d(p)$, defined by

$$
\left\langle(-D \partial)^{-1}\right\rangle=\frac{d(p)}{p^{2}},
$$

obtained in the variational approach and on the lattice. In the variational approach one can show that the infrared exponents of the ghost and gluon propagators,

$$
\omega(p \rightarrow 0) \sim 1 / p^{\alpha}, \quad d(p \rightarrow 0) \sim 1 / p^{\beta},
$$




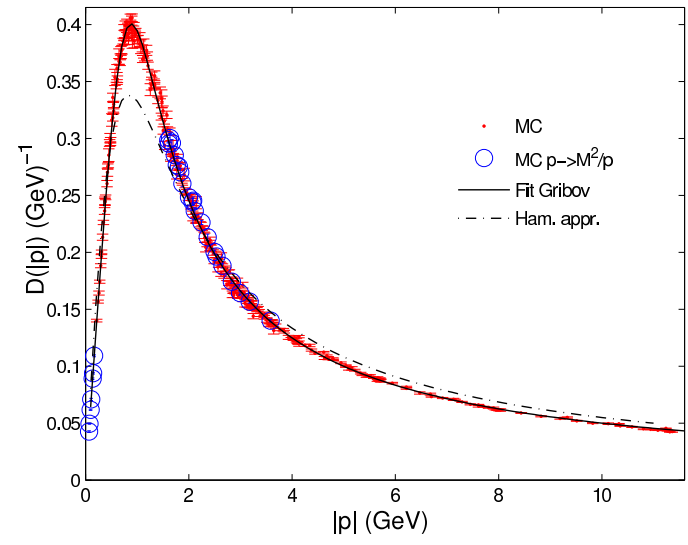

Figure 1: Gluon propagator from the variational approach compared to lattice data

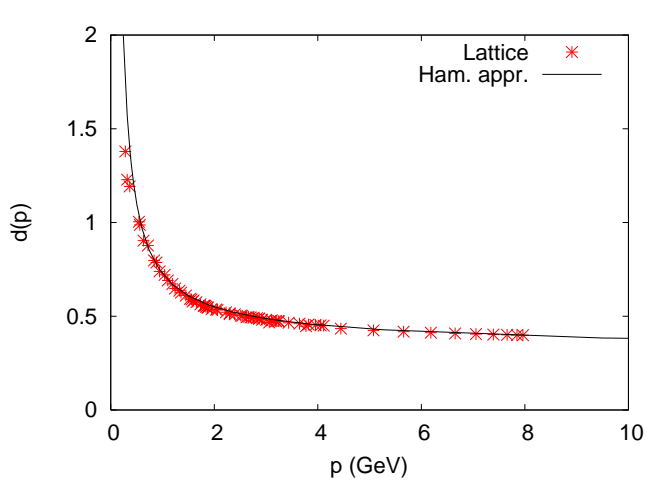

Figure 2: Ghost form factor from the variational approach compared to lattice data

are related by a sum rule under the assumption of a trivial scaling of the ghost-gluon vertex [7],

$$
\alpha=2 \beta-1 .
$$

Furthermore, assuming an infrared scaling behaviour of the ghost form factor, i.e. $\beta>0$, one finds two "critical" solutions

$$
\text { i) } \quad \alpha=0.6, \quad \beta=0.8 \quad \text { ii) } \quad \alpha=1, \quad \beta=1 .
$$

The latter results in a linearly rising quark potential shown in Fig. 3. However, sub-critical solutions $d^{-1}(0) \neq 0$ do also exist [8]. Previous lattice calculations in Coulomb gauge seemed to yield $\beta=\frac{1}{2}$. However, these calculations were carried out on rather small lattices. As one increases the lattice size, $\beta$ seems to increase as well and recent lattice calculations [6] are at least compatible with $\beta=1$. In Coulomb gauge the inverse ghost form factor has been shown to represent the dielectric function of the Yang-Mills vacuum [9], $\varepsilon(p)=d^{-1}(p)$, and the so-called horizon condition $d^{-1}(0)=0$ implies then that the Yang-Mills vacuum is a perfect dual color superconductor.

The advantage of the Hamiltonian formulation is its close connection to physics. In the variational approach one makes an ansatz for the unknown vacuum wave functional which encodes all the physics. This ansatz can be systematically improved towards the full theory. The price to pay is the apparent loss of renormalization group invariance which already complicates the discussion of perturbative renormalization, but also the discussion of scaling laws in the infrared.

\section{FRG flow equation}

Renormalization group invariance is naturally built-in in the functional renormalization group approach to the Hamilton formulation of Yang-Mills theory which hence has the advantage of combining renormalization group invariance with the physical Hamiltonian picture.

The starting point of the FRG flow equation approach [2] is the (renormalized) generating functional of Green's functions

$$
Z[j]=\int \mathscr{D} \varphi e^{-S[\varphi]+j \cdot \varphi},
$$


where $\varphi$ and $j$ denote collectively all fields and sources. In the FRG approach $Z[j]$ is infrared regulated by adding the regulator term

$$
\Delta S_{k}[\varphi]=\frac{1}{2} \varphi \cdot R_{k} \cdot \varphi \equiv \frac{1}{2} \int \varphi R_{k} \varphi
$$

to the classical action,

$$
Z_{k}[j]=\int \mathscr{D} \varphi e^{-S[\varphi]-\Delta S_{k}[\varphi]+j \cdot \varphi} \equiv e^{W_{k}[j]} .
$$

The regulator function $R_{k}(p)$ is an effective momentum dependent mass with the properties

$$
\lim _{p / k \rightarrow 0} R_{k}(p)>0, \lim _{k / p \rightarrow 0} R_{k}(p)=0,
$$

which ensures that $R_{k}(p)$ suppresses propagation of modes with $p \lesssim k$ while those with $p \gtrsim k$ are unaffected and the full theory at hand is recovered as the cut-off scale $k$ is pushed to zero.

By taking the derivative of Eq. (3.3) with respect to the (dimensionless) momentum cut-off $t=\ln k / k_{0}$, one obtains the flow equation for the generating functional of connected Green's functions

$$
\partial_{t} W_{k}[j]=-\frac{1}{2} \frac{\delta W_{k}}{\delta j} \dot{R}_{k} \frac{\delta W_{k}}{\delta j}-\frac{1}{2} \operatorname{Tr} \dot{R}_{k} \frac{\delta^{2} W_{k}}{\delta j \delta j}
$$

(where the dot denotes $\partial_{t}$ ). In practice, it is usually more convenient to perform first the Legendre transform from the source $j$ to the classical field

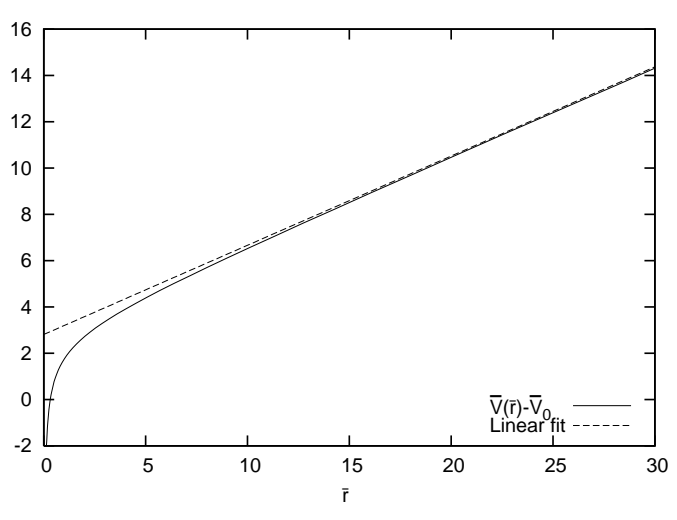

Figure 3: Static quark potential

$$
\phi=\frac{\delta W_{k}[j]}{\delta j}
$$

resulting in the effective action

$$
\Gamma_{k}[\phi]=\left(-W_{k}[j]+j \cdot \phi\right)_{j=j_{k}[\phi]}-\frac{1}{2} \phi \cdot R_{k} \cdot \phi,
$$

where $j_{k}[\phi]$ is given by solving Eq. (3.6) for $j$. By taking the derivative of Eq. (3.7) w.r.t. $t=\ln k / k_{0}$ and using Eq. (3.5) one derives Wetterich's flow equation for $\Gamma_{k}$,

$$
\partial_{t} \Gamma_{k}[\phi]=\frac{1}{2} \operatorname{Tr} \frac{1}{\Gamma_{k}^{(2)}[\phi]+R_{k}} \dot{R}_{k}
$$

where

$$
\Gamma_{k, 1 \ldots n}^{(n)}[\phi]=\frac{\delta^{n} \Gamma_{k}[\phi]}{\delta \phi_{1} \ldots \delta \phi_{n}}
$$

are the one-particle irreducible $n$-point functions (proper vertices). The generic structure of the flow equation (3.8) is independent of the details of the underlying theory, i.e., of the explicit form of the action $S[\phi]$, but is a mere consequence of the form of the regulator term (3.2), i.e., that it is 
quadratic in the field. By taking functional derivatives of Eq. (3.8) one obtains the flow equations for the (inverse) propagators and proper vertices. For the two-point function this equation reads

$$
\partial_{t} \Gamma_{k, 12}^{(2)}=\frac{1}{2} \operatorname{Tr} \dot{R}_{k} \frac{1}{\Gamma_{k}^{(2)}+R_{k}}\left\{-\Gamma_{k, 12}^{(4)}+\left[\Gamma_{k, 1}^{(3)} \frac{1}{\Gamma_{k}^{(2)}+R_{k}} \Gamma_{k, 2}^{(3)}+(1 \leftrightarrow 2)\right]\right\} \frac{1}{\Gamma_{k}^{(2)}+R_{k}},
$$

where all cyclic indices (summed over in the trace) have been suppressed.

\section{Hamiltonian flow}

In the Hamiltonian approach the generating functional of static correlation functions reads

$$
Z[j]=\langle\psi|\exp (j \cdot \varphi)| \psi\rangle=\int \mathscr{D} \varphi|\psi[\varphi]|^{2} \exp (j \cdot \varphi),
$$

where $\langle\varphi \mid \psi\rangle=\psi[\varphi]$ is the vacuum wave functional. With the identification

$$
|\psi[\varphi]|^{2} \equiv \exp (-S[\varphi]),
$$

defining the classical action $S[\varphi]$ in terms of the vacuum wave functional $\psi[\varphi]$, Eq. (4.1) has precisely the standard form of a generating functional except that the functional integral extends over time independent fields. Here we are interested in the Hamiltonian flow of Yang-Mills theory in Coulomb gauge whose generating functional reads

$$
Z[J]=\int \mathscr{D} A \operatorname{Det}(-D \partial)|\psi[A]|^{2} \exp (J \cdot A),
$$

where the integration is over transversal gauge fields $A$ and the Coulomb gauge condition has been implemented by the usual Faddeev-Popov method. Representing the Faddev-Popov determinant in the standard fashion by ghost fields, $c, \bar{c}$,

$$
\operatorname{Det}(-D \partial)=\int \mathscr{D} \bar{c} \mathscr{D} c e^{-\int \bar{c}(-D \partial) c}
$$

the underlying action, cf. Eq. (4.2), reads

$$
S[\varphi]=-\ln |\psi[A]|+\int \bar{c}(-D \partial) c .
$$

The general flow equation (3.10) still holds provided that $\phi$ is interpreted as the superfield $\phi=$ $(A, c, \bar{c})$. The FRG flow equations for the gluon and ghost propagators are diagrammatically given in Fig. 4.

\section{Approximation schemes and numerical solution}

The FRG flow equations embody an infinite tower of coupled equations for the flow of the propagators and the proper vertices. These equations have to be truncated to get a closed system. We shall use the following truncation: we only keep the gluon and ghost propagators, to wit

$$
\Gamma_{k, A A}^{(2)}=2 \omega_{k}(p), \quad \quad \Gamma_{k, \bar{c} c}^{(2)}=\frac{p^{2}}{d_{k}(p)},
$$

In addition, we keep the ghost-gluon vertex $\Gamma_{k, A \bar{c} c}^{(3)}$, which we assume to be bare, i.e., we do not solve its FRG flow equation. The above truncation removes the tadpole diagrams from Fig. 4. Moreover, we shall assume infrared ghost dominance and discard gluon loops. Then the flow equations of the ghost and gluon propagator reduce to the ones shown in Fig. 5. 


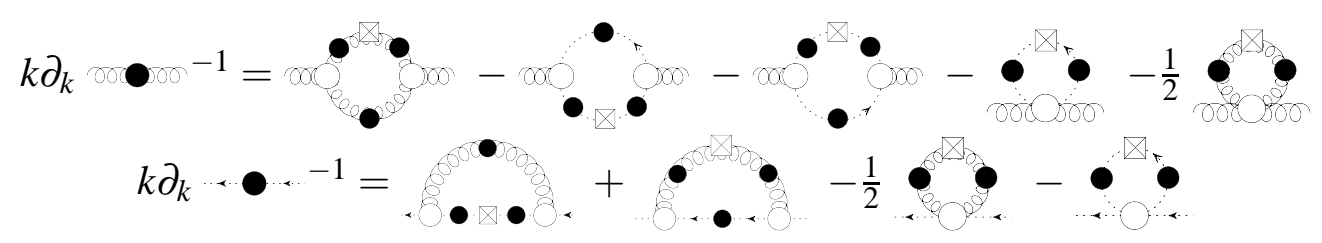

Figure 4: Flow equations of the propagators. The spiral and dotted lines with black circles denote the regularized gluon and ghost propagators at cutoff momentum $k$, respectively. White circles stand for proper vertices at cutoff $k$, a regulator insertion $\dot{R}_{k}$ is represented by a square with a cross.

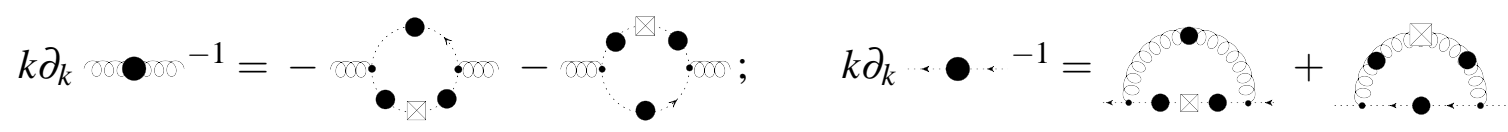

Figure 5: Truncated propagator flow equations. The bare vertices at $k=\Lambda$ are symbolized by small dots.

The flow equations in Fig. 5 are solved numerically using the regulators

$$
\begin{aligned}
& R_{A, k}(p)=2 p r_{k}(p), R_{c, k}(p)=p^{2} r_{k}(p), \\
& r_{k}(p)=\exp \left[\frac{k^{2}}{p^{2}}-\frac{p^{2}}{k^{2}}\right]
\end{aligned}
$$

and the perturbative initial conditions at the large momentum scale $k=\Lambda$,

$$
d_{\Lambda}(p)=d_{\Lambda}=\text { const. }, \quad \omega_{\Lambda}(p)=p+a .
$$

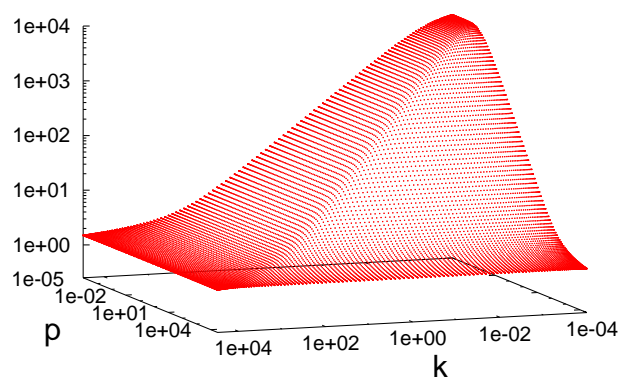

Figure 6: Flow $d_{k}(p)$ of the ghost form factor.

With these initial conditions, the flow equations for the ghost and gluon propagators are solved under the constraint of infrared scaling for the ghost form factor. The resulting full flow of the ghost dressing function is shown in Fig. 6. As the IR cut-off momentum $k$ is decreased, the ghost form factor $d_{k}(p)$ (constant at $k=\Lambda$ ) builds up infrared strength and the final solution at $k=k_{\min }$ is shown in Fig. 7 together with the one for the gluon energy $\omega_{k_{\min }}(p)$. It is seen that the IR exponents, i.e., the slopes of the curves $d_{k_{\min }}(p), \omega_{k_{\min }}(p)$ do not change as the minimal cut-off $k_{\min }$ is lowered. Let us stress that we have assumed infrared scaling of the ghost form factor but not the horizon condition. The latter was obtained from the integration of the flow equation but not put in by hand (the same is also true for the infrared analysis of the Dyson-Schwinger equations following from the variational Hamiltonian approach, i.e., assuming scaling the DSEs yield the horizon condition). The infrared exponents extracted from the numerical solutions of the flow equations are

$$
\alpha=0.28, \quad \beta=0.64 \text {. }
$$

They satisfy the sum rule (2.6) resulting from the DSE obtained in the variational approach but are smaller than the ones of the DSE, see Eq. (2.7). Moreover, the present approach allows to prove the uniqueness of the sum rule (2.6) [1], analogously to the proof in Landau gauge [10].

Replacing the propagators with running cut-off momentum scale $k$ under the loop integrals of the flow equation by the propagators of the full theory,

$$
d_{k}(p) \rightarrow d_{k=0}(p), \omega_{k}(p) \rightarrow \omega_{k=0}(p),
$$



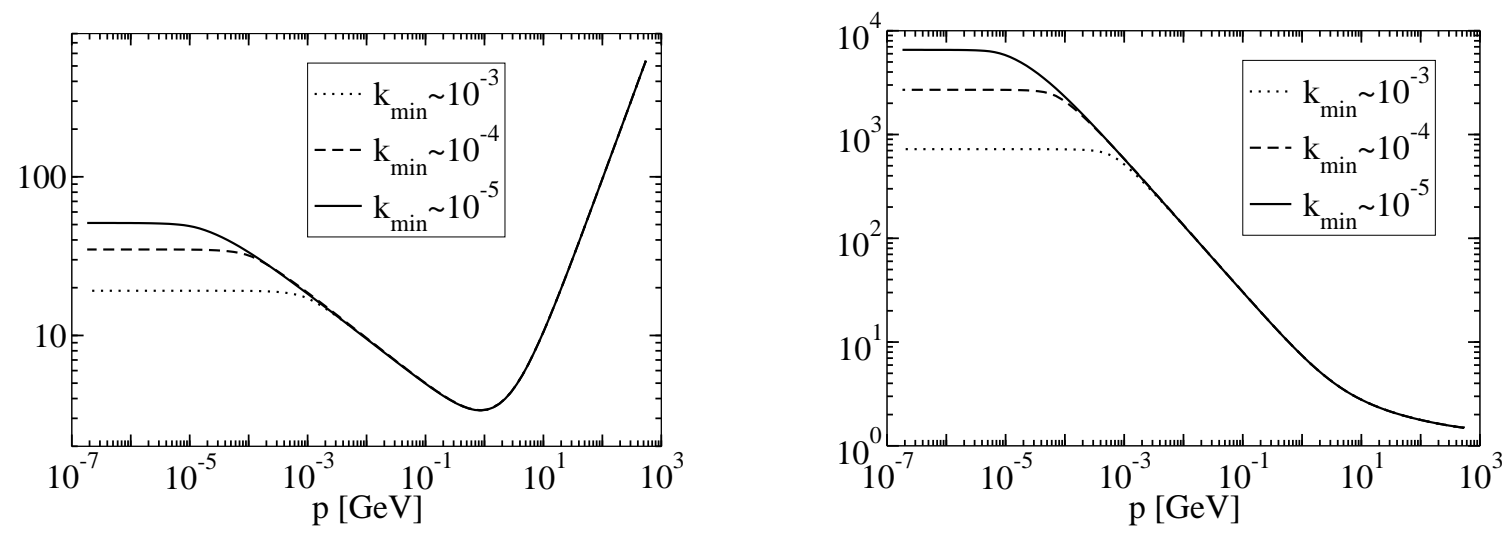

Figure 7: Inverse gluon propagator $\omega$ and ghost form factor $d$ at three minimal cutoff values $k_{\min }$.

amounts to taking into account the tadpole diagrams [1]. Then the flow equations can be analytically integrated and turn precisely into the DSEs obtained in the variational approach to the Hamiltonian formulation of Yang-Mills theory [4], with explicit UV regularization by subtraction. This establishes the connection between these two approaches and highlights the inclusion of a consistent UV renormalization procedure in the present approach.

The above results encourage further studies, which includes the flow of the potential between static color sources as well as dynamic quarks.

\section{References}

[1] M. Leder, J. M. Pawlowski, H. Reinhardt and A. Weber, arXiv:1006.5710 [hep-th].

[2] D. F. Litim and J. M. Pawlowski, arXiv:hep-th/9901063; J. M. Pawlowski, Annals Phys. 322, 2831 (2007) [arXiv:hep-th/0512261]; H. Gies, arXiv:hep-ph/0611146.

[3] A. P. Szczepaniak and E. S. Swanson, Phys. Rev. D 65, 025012 (2002) [arXiv:hep-ph/0107078]. A. P. Szczepaniak, Phys. Rev. D 69, 074031 (2004) [arXiv:hep-ph/0306030].

[4] C. Feuchter and H. Reinhardt, Phys. Rev. D 70, 105021 (2004) [arXiv:hep-th/0408236]. H. Reinhardt and C. Feuchter, Phys. Rev. D 71, 105002 (2005) [arXiv:hep-th/0408237].

[5] D. Epple, H. Reinhardt and W. Schleifenbaum, Phys. Rev. D 75, 045011 (2007) [arXiv:hep-th/0612241].

[6] G. Burgio, M. Quandt and H. Reinhardt, Phys. Rev. D 81, 074502 (2010) [arXiv:0911.5101 [hep-lat]].

[7] W. Schleifenbaum, M. Leder and H. Reinhardt, Phys. Rev. D 73, 125019 (2006) [arXiv:hep-th/0605115].

[8] D. Epple, H. Reinhardt, W. Schleifenbaum and A. P. Szczepaniak, Phys. Rev. D 77, 085007 (2008) [arXiv:0712.3694 [hep-th]].

[9] H. Reinhardt, Phys. Rev. Lett. 101 (2008) 061602 [arXiv:0803.0504 [hep-th]].

[10] C. S. Fischer and J. M. Pawlowski, Phys. Rev. D 80 (2009) 025023 [arXiv:0903.2193 [hep-th]]; Phys. Rev. D 75 (2007) 025012 [arXiv:hep-th/0609009]. 